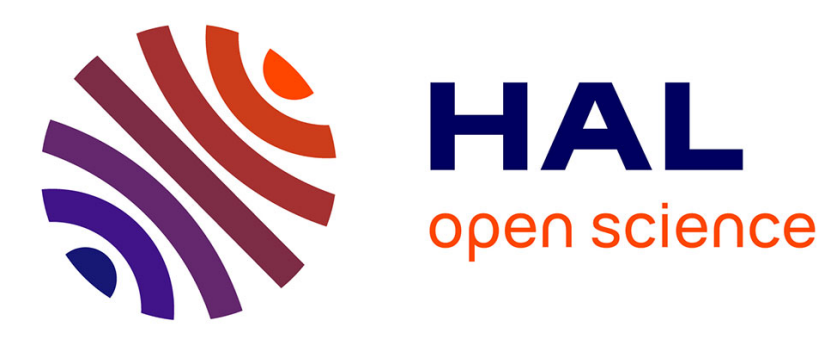

\title{
Sub-hour Unit Commitment MILP Model with Benchmark Problem Instances
}

Paula Carroll, Damian Flynn, Bernard Fortz, Alex Melhorn

\section{To cite this version:}

Paula Carroll, Damian Flynn, Bernard Fortz, Alex Melhorn. Sub-hour Unit Commitment MILP Model with Benchmark Problem Instances. ICCSA 2017 - 17th International Conference on Computational Science and Its Applications, Jul 2017, Trieste, Italy. pp.635-651, 10.1007/978-3-319-62395-5_44 . hal-01665611

\section{HAL Id: hal-01665611 \\ https://hal.inria.fr/hal-01665611}

Submitted on 18 Dec 2017

HAL is a multi-disciplinary open access archive for the deposit and dissemination of scientific research documents, whether they are published or not. The documents may come from teaching and research institutions in France or abroad, or from public or private research centers.
L'archive ouverte pluridisciplinaire HAL, est destinée au dépôt et à la diffusion de documents scientifiques de niveau recherche, publiés ou non, émanant des établissements d'enseignement et de recherche français ou étrangers, des laboratoires publics ou privés.

$$
\text { Copyright }
$$




\title{
Sub-hour Unit Commitment MILP Model with Benchmark Problem Instances
}

\author{
Paula Carroll ${ }^{1,2}$, Damian Flynn ${ }^{2}$, Bernard Fortz ${ }^{3}$, and Alex Melhorn ${ }^{2}$ \\ 1 Centre for Business Analytics, University College Dublin, Ireland \\ ${ }^{2}$ Electricity Research Centre, University College Dublin, Ireland \\ 3 Département d'informatique, Univeristé Libre de Bruxelles, Belgium and INOCS, \\ INRIA Lille Nord-Europe, France \\ paula.carroll@ucd.ie
}

\begin{abstract}
Power systems are operated to deliver electricity at minimum cost while adhering to operational and technical constraints. The introduction of smart grid technologies and renewable energy sources offers new challenges and opportunities for the efficient and reliable management of the grid. In this paper we focus on a Mixed Integer Programming sub-hour Unit Commitment model. We present analysis of computational results from a large set of problem instances based on the Irish system and show that problem instances with higher variability in net demand (after the integration of renewables) are more challenging to solve.
\end{abstract}

\section{Integrating Renewable Energy Sources (RESs)}

The Irish Government is aiming for $40 \%$ of electricity to be generated from renewable energy sources (RESs) by 2020 in response to an EU directive. Ireland is rich in wind resources but integrating wind energy creates new operational and planning challenges for Transmission System Operators (TSOs).

In a deregulated market the TSO plays a central role in determining which generating units should be committed to meet estimated demand. The classical Unit Commitment (UC) problem determines which generators to start up (shut down) on a day-ahead scheduling basis. The demand to be met by traditional thermal generators can be estimated as the forecast load. This gross load can be offset by the power available from RESs and demand response. This approach can lead to net demand load patterns that are quite different to the typical diurnal electricity demand pattern. An example of the typical diurnal demand pattern is shown in Figure 1a. The more variable net load instances such as that in Figure 1b prove more computationally challenging than instances exhibiting the traditional diurnal pattern.

The need for more detailed UC models is addressed in [23]. Operation of the system at sub-hourly levels offers increased flexibility [15,24], but leads to computational challenges for mixed integer linear programming (MILP) models. Using MILP models to solve continuous time problems leads to issues of discretisation. We need to adjust the models to cater for the finer time step granularity. These 


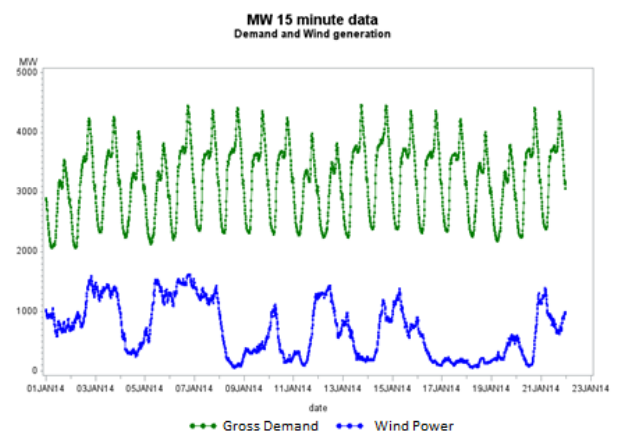

(a) Gross Demand and Wind Power

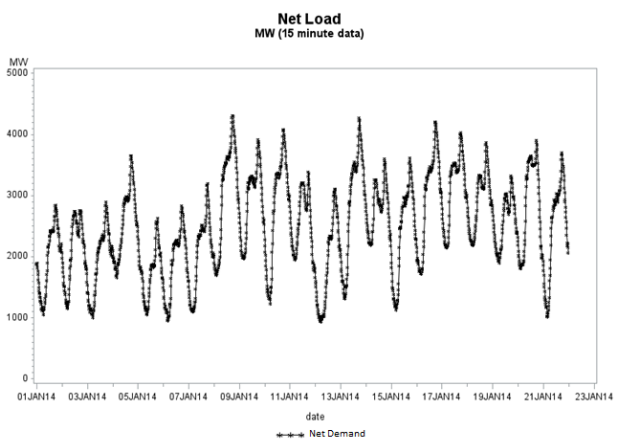

(b) Net Demand Example

Fig. 1: Comparison of Gross and Net demand instances, Ireland January 2014.

challenges provide new opportunities for the business analytics and optimisation communities to design efficient solution approaches.

The contributions of this paper are a detailed sub-hourly UC MILP model and a set of insights gained from computational experiments on realistic UC test instances based on the Irish system. Our analysis gives some insight into what makes a UC instance more difficult to solve.

\section{The Unit Commitment Problem}

We focus on the Thermal Unit Commitment problem. i.e., conventional thermal generating units where fuel is converted to produce electric power. The UC problem can be stated as follows:

\section{Instance:}

- a set of thermal generating units (GU) $G$ and their operating characteristics,

- a set of load demands $D$ and required reserves $R$ per time step $k$ over a planning horizon of $K$ periods. In our case, a set of wind energy levels are also given.

\section{Problem Statement:}

Determine the minimum cost GU dispatch schedule that meets forecast demand and satisfies GU operating characteristics.

We consider a planning horizon of a single day. In practice UC MILP models are solved by the TSO for the day-ahead market with subsequent in-day updates and real time adjustments. Estimated demand must be met. Hydro, interconnect, renewable energy and demand side interventions can be reflected as a simple reduction in demand. In this paper focusing on the integration of wind energy, the amount of demand to be met by thermal generation $D$ can be reduced by the amount of wind power available. 
Reserve power in the system is specified in case of failures or outages. The higher the reserve value, the better the operational security, but at a higher cost. In much of the literature a simple $10 \%$ reserve rule is suggested, which means that demand is effectively inflated by $10 \%$. Blackouts in recent years have increased the focus on the design and operation of secure grids [4] and $n-1$ constraint ensure the reserve must be equal to or greater than the largest generator online. In the case of systems with significant utilisation of RESs, the variable nature of the source leads to additional focus on forecasting techniques to quantify reserve requirements and to reduce the supply side forecast error. See for example [10].

In addition, there is increased interest in algorithmic techniques that address the data uncertainty in energy problems. Stochastic programming and robust optimisation techniques have been applied to various aspects of power grid operations. See for example $[2,18,25]$.

The focus of this paper is on a more detailed unit commitment model which is of practical interest as electricity markets migrate to sub-hourly operation to facilitate the integration of renewable energy sources, demand response and smart grid initiatives.

Each GU $g \in G$ has a set of operating characteristics specified as:

$\bar{P}_{g} \quad$ Maximum output $(\mathrm{MW})$

$\underline{P}_{g} \quad$ Minimum output (MW)

$U T_{g}$ Minimum time that a unit must stay online (up) once it has been switched online

$D T_{g} \quad$ Minimum time that a unit must stay offline (down) once it has been switched offline

$I S_{g} \quad$ Initial State, number of time steps unit on (off) line at $k=0$

$a_{g}, b_{g}, c_{g}$ Coefficients of quadratic power production cost function

$c_{g}, c c_{g}$ Hot (cold) start cost coefficients

$t_{g}^{\text {cold }}$ Number of time steps for unit to cool fully (after min down time)

GU power production costs are described by quadratic functions. MILP approaches can be used in conjunction with piecewise approximations to solve UC instances. There are many additional GU parameters that may be considered, such as:

ramp-up/down $\left(R U_{g}, R D_{g}\right)$ limits: rate of power change when running start-up/shut-down $\left(S U_{g}, S D_{g}\right)$ limits: rate of power change at start up/shut down

shut-down costs, $C_{g}^{d}$ : the cost of lost fuel

Further variants of the basic UC problem may also include power output at start of planning horizon, final state requirements, operational requirements, maintenance schedules, cycling constraints, fuel usage constraints, plant crew considerations, emission constraints $\left(\mathrm{CO}_{2}, \mathrm{NO}_{x}\right.$, and $\left.\mathrm{SO}_{x}\right)$, reserve constraints to ensure security (Simple rule $(x \%)$, Contingency or Control) and network or transmission constraints. 
A review of UC solution approaches is given in [19]. Approaches include dynamic programming (DP), Lagrangian relaxation (LR), MILP, simulated annealing (SA), expert systems and artificial neural networks, fuzzy systems, genetic algorithms (GA), evolutionary programming (EP), ant colony heuristics, particle swarm optimisation and hybrid approaches. In many cases the test systems are not fully described making reproduction and comparison of the empirical results difficult. Table 1 gives a summary of highly cited UC solution approaches.

Table 1: UC Solution Approaches

\begin{tabular}{|c|c|c|c|}
\hline Reference & Year & Approach & Test data \\
\hline [14] & 1996 & GA & 10 base units, no ramping rates or shutdown costs \\
\hline$[5]$ & 2006 & MILP & $10-100$ units based on $[14]$ \\
\hline [27] & 1988 & LR & 100 units, details not available \\
\hline [13] & 1999 & $\mathrm{EP}$ & 10 - 100 units based on [14] \\
\hline$[17]$ & 1983 & $\mathrm{LR}$ & 172 units, details not available \\
\hline$[7]$ & 2000 & $\mathrm{LR}, \mathrm{GA}$ & 10 - 100 units based on [14] \\
\hline [21] & 1996 & $\mathrm{SP}$ & not specified \\
\hline [20] & 1987 & $\mathrm{DP}$ & not specified \\
\hline [26] & 1990 & $\mathrm{SA}$ & 10 and 100 units, details not available \\
\hline$[9]$ & 1978 & $\mathrm{SP}$ & 5 units, details given \\
\hline [8] & 1983 & MILP & not specified \\
\hline [25] & 2009 & MILP & 45 unit test system, details not available \\
\hline$[22]$ & 2006 & PSO & 10 units from [14] \\
\hline
\end{tabular}

\section{Unit Commitment (sub-hour) MILP model}

A UC model with ramping constraints is described in [5]. This paper presents a subhour variant of the UC MILP model with ramping constraints. We include the following ideas:

1. A set of real variables are introduced to simplify the implementation of hot and cold start costs;

2. A set of start up and shut down variables are introduced to allow a slow unit to start up or shut down over a number of time steps. This is important in subhour models with finer time step granularity;

3. The ramp (start/shutdown) constraints are adapted for slow units and subhourly models;

Let $g \in G$ be the index of each generating unit, let $k \in K$ be the index of each time step. Let $c_{g, k}^{p}, c_{g, k}^{u}, c_{g, k}^{d} \in \mathbb{R}^{+}$be sets of decision variables representing the power production, start-up and shut-down costs respectively. Let $p_{g, k}$ and $\bar{p}_{g, k} \in \mathbb{R}^{+}$be the power output and power availability variables. $v_{g, k}$ are binary variables set to 1 if unit $g$ is on, zero otherwise. $\delta_{g, k, l}, l \in L$ are the variables of a delta-approach to a piecewise linear approximation of $c_{g, k}^{p}$ of $L$ line segments. 
With these sets of variables, a UC model can be formulated as follows:

$$
\begin{array}{lll}
\min & \sum_{k \in K} \sum_{g \in G} c_{g, k}^{p}+c_{g, k}^{u}+c_{g, k}^{d} & \\
\text { s.t. } & \sum_{g \in G} p_{g, k}=D_{k} & \\
& \sum_{g \in G} \bar{p}_{g, k} \geq D_{k}+R_{k} & \forall k \in K \\
& \underline{P}_{g} \cdot v_{g, k} \leq p_{g, k} \leq \bar{p}_{g, k} & \forall g \in G, k \in K \\
& \bar{p}_{g, k} \leq \bar{P}_{g} \cdot v_{g, k} & \forall g \in G, k \in K \\
& c_{g, k}^{p}=a_{g}+b_{g} \cdot p_{g, k}+c \cdot p_{g, k}^{2} & \forall g \in G, k \in K
\end{array}
$$

Objective The objective is to minimise operating costs over a planning horizon, usually a (rolling) daily horizon. Traditionally power production, start-up and shut-down costs of each generator are included. Work is ongoing on how to best capture the cost of RESs, reserve, cycling and emissions in the objective function. Note that the objective function in this model does not explicitly charge for reserve, i.e., only $p$ appears in the objective, not $\bar{p}$.

Production Constraints The primary constraint is the production constraint (2). Total production of the units at a given time must equal the demand. An approach to integrating RESs, is to reduce the demand target by the amount of renewable capacity predicted to be available to give net demand.

Reserve constraints (3) ensure that the maximum production available meets the additional reserve target. Constraints (4) and (5) ensure the production of an individual unit lies between its minimum and maximum output when online.

Production Cost The quadratic power production cost in (6) is usually approximated by a piecewise linear (PWL) approximation. See $[1,5,12,23]$. The following is a delta approach PWL approximation of $L$ line segments:

$$
\begin{array}{ll}
c_{g, k}^{p}=A_{g} \cdot v_{g, k}+\sum_{l \in L} F_{l, g} \cdot \delta_{l, g, k} & \forall g \in G, k \in K \\
p_{g, k}=\underline{P}_{g} \cdot v_{g, k}+\sum_{l \in L} \delta_{l, g, k} & \forall g \in G, k \in K \\
\delta_{1, g, k} \leq T_{l, g}-\underline{P}_{g} & \forall g \in G, k \in K \\
\delta_{l, g, k} \leq T_{l, g}-T_{l-1, g} & \forall g \in G, k \in K, l \in L \backslash 1
\end{array}
$$

where $A_{g}$ is the no-load cost given by: $A_{g}=a_{g}+b_{g} \cdot \underline{P}_{g}+c_{g} \cdot \underline{P}_{g}{ }^{2} \quad \forall \quad g \in G$, $F_{l, g}$ is the slope of line segment $l \in L$, and $T_{l, g}$ are the breakpoints of the power intervals from $\underline{P}_{g}$ to $\bar{P}_{g}$. Note in this implementation $T_{L, g}=\bar{P}_{g} \quad \forall \quad g \in G$. 
Minimum up and Down times Unit $g$ is required to stay on initially for at least $G_{g}:=\min \left(K, \max \left(\left(U T_{j}-I S_{g}\right) v_{g, 0}, 0\right)\right)$ steps once turned on. Variables $v_{g, k}$ can be fixed for the required number of steps for units that must be kept on initially.

During the operating horizon the minimum up constraints are given by:

$$
\sum_{n=k}^{k+U T_{g}-1} v_{g, n} \geq U T_{g}\left(v_{g, k}-v_{g, k-1}\right) \quad \forall g \in G, k=G_{g}+1 \ldots K-U T_{g}+1
$$

Minimum up constraints for the final steps of the horizon are:

$$
\sum_{n=k}^{K}\left(v_{g, n}-\left(v_{g, k}-v_{g, k-1}\right)\right) \geq 0 \quad \forall g \in G, k=K-U T_{g}+2 \ldots K
$$

Similarly, (13) and (14) enforce a minimum down time of $L_{g}$ time steps when unit $g$ is switched off where $\left.L_{g}=\min \left(K, \min \left(D T_{j}+I S_{g}\right)\left(1-v_{g, 0}\right), 0\right)\right)$.

$$
\begin{array}{ll}
\sum_{n=k}^{k+D T_{g}-1}\left(1-v_{g, n}\right) \geq D T_{g}\left(v_{g, k-1}-v_{g, k}\right) & \forall g \in G, k=L_{g}+1 \ldots T-D T_{g}+1 \\
\sum_{n=k}^{T}\left(1-v_{g, n}-\left(v_{g, k-1}-v_{g, k}\right)\right) \geq 0 & \forall g \in G, k=T-D T_{g}+2 \ldots T
\end{array}
$$

Minimum up and down constraints (11) and (13) can be strengthened by disaggregation, but our experience shows that it decreases the performance of the solver as it considerably increases the size of the model.

\subsection{Slow and sub-hour ramping}

UC models such as [5] allow a unit to turn on if it can ramp up to $\underline{P}_{g}$ in a single time step. Likewise, a unit can only be turned off if it can do so in a single step. This results in unrealistic solutions that reflect the problem instance initial status. A unit with slow start-up or shut-down rates may need to be turned on (off) and allowed to start-up (shut-down) over a series of steps. This issue becomes a particular concern when operating at a sub-hourly resolution when even fast units may require a number of sub-hourly time steps to reach operating power limits.

A $\operatorname{Big} M$ approach is used in [5] to model the ramping constraints. As an alternative approach to the ramping constraints in [5], we introduce additional binary variables $u$ and $w \in\{0,1\}$ a unit can be allowed to turn on (shut down) over a series of steps before (after) the unit is in the synchronised production state as shown in Figure 2. Power generated during the start up and shut down phases can be used to satisfy demand but stable system operation is only ensured when the production power for a unit that is on is maintained within its generation limit bounds, $\underline{P}_{g, k}$ and $\bar{P}_{g, k}$. The constraints below ensure that a unit is started up (shut down) as quickly as possible. The costs of power produced 
during the starting up and shut down phases are captured in the start up and shut down fixed costs respectively.

The minimum number of time steps required for a unit to start up is calculated as $S U T_{g}=\left\lfloor\underline{P}_{g} / S U_{g}\right\rfloor$. Similarly, the minimum number of time steps for a unit to shut down is $S D T_{g}=\left\lfloor\underline{P}_{g} / S D_{g}\right\rfloor$. This allows a unit to be brought just below $P_{g}$ at time step $k$ by starting up at the maximum startup rate. It is then ready to breach $P_{q}$ at or below the ramp up rate in step $k+1$.

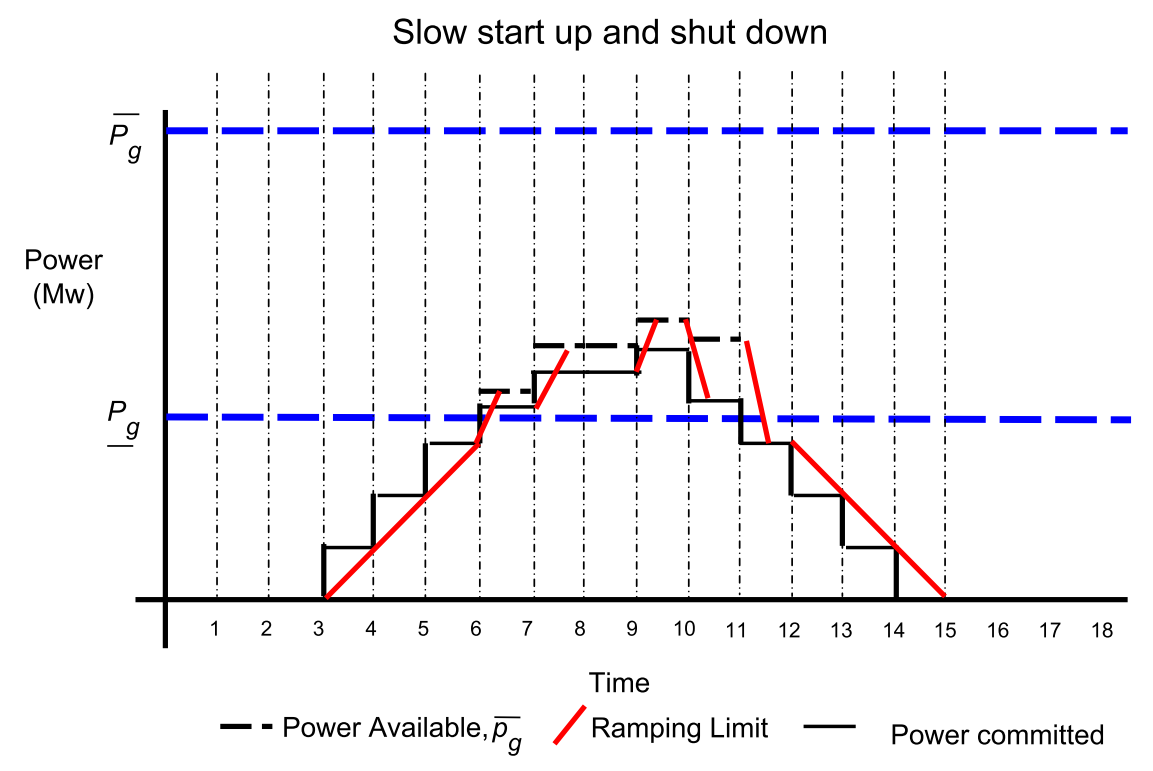

Fig. 2: Slow/sub-hour ramping restrictions.

Likewise, a unit can be brought from just above $P_{g}$ at time $k-1$ to below $P_{g}$ in time step $k$ and shut down in $S D T_{g}$ steps at the maximum shut down rate. The available power during normal operations is still restricted to within the unit's operating limits. However using this approach, a small amount of additional power is available during a start up or shut down. Constraints (2) can be modified to:

$$
\sum_{g \in G} p_{g, k}+S U_{g}\left(\sum_{l=\min \left(1, k-S U T_{g}+1\right)}^{k} u_{g, k}\right)+S D_{g}\left(\sum_{l=k}^{\min \left(k+S D T_{g}-1, K\right)} w_{g, k}\right)=D_{k} \quad \forall k \in K
$$

The amount of power during the start up or shut down phase is bounded by the minimum power threshold $\underline{P}_{g}$. If a unit is on at time $k$, the power available from start up completion at $k-1$ or start of shut down at $k+1$ is bounded by $\underline{P}_{g}$.

$$
S U_{g}\left(\sum_{l=k-S U T_{g}}^{k-1} u_{g, l}\right)+S D_{g}\left(\sum_{l=k+1}^{k+S D T_{g}} w_{g, l}\right) \leq \underline{P}_{g} v_{g, k} \quad \forall g \in G, k \in K
$$


Fast units can start or shut down in a single step. In such cases $u$ or $w$ variables are not required. The $u$ and $v$ variables for slow units are linked as follows:

$$
u_{g, k-l}+v_{g, k-1} \geq v_{g, k} \quad \forall g \in G, k \in K \mid k-S U T_{g} \geq 1,1 \leq l \leq S U T_{g}
$$

Constraints (16) force a slow unit to begin starting up for SUT steps prior to $k$ if the unit switches on in step $k$.

The following constraints ensure a slow unit enters the production state as soon as possible if it is started up:

$$
v_{g, k} \geq u_{g, k-S U T}-u_{g, k-S U T-1} \quad \forall g \in G, k \in K \mid k-S U T_{g}-1 \geq 1
$$

A similar constraint is added for the initial time steps $S U T-1<0$ based on the unit's initial status.

Ramping Up Figure 2 shows the ramp limits from one time step to the next. Ramp up constraints give an upper bound on the difference between $\bar{p}_{g, k}$ and $p_{g, k-1}$. Ramp down constraints give a lower bound for the difference with $p_{g, k+1}$. Ramp up constraints for slow units can be expressed as:

$$
\bar{p}_{g, k} \leq p_{g, k-1}+S U_{g} S U T_{g}\left(u_{g, k-1}-u_{g, k}\right)+R U_{g}\left(v_{g, k}\right) \quad \forall g \in G, k \in K \backslash\{1\}
$$

If a unit starts switching on at $k-S U T_{g}$, it continues switching on up to $k-1$, because $u_{g, k-1}=1$ by (16). The $O n$ status of the unit is reached after $S U T_{g}$ steps when $v_{g, k}$ goes to 1 and $u_{g, k}$ goes to 0 . The power available in step $k$ is the $S U_{g}$ term of (18) plus some ramp up in step $k$ (bounded by $R U_{g}$ ).

If the unit is already on and was not started in step $k-1\left(u_{g, k-1}=0\right)$, then the $u$ terms are zero and the power available in $k$ can increase from the power output in the previous step plus an amount up to the ramp-up rate $R U_{g}$. This is captured in the $R U_{g}$ term of (18).

The equivalent ramp up constraint for a fast start unit is:

$$
\bar{p}_{g, k} \leq p_{g, k-1}+S U_{g}\left(v_{g, k}-v_{g, k-1}\right)+R U_{g} v_{g, k-1} \quad \forall g \in G, k \in K \backslash\{1\}
$$

The power available in the first time step needs to be handled separately and is dependent on the initial conditions of the system.

Constraints (19) ensure that the system is capable of ramping up to $\bar{p}_{g, k}$ from $p_{g, k-1}$ in a single time step. In general, the system deploys an amount of power equal to $p_{g, k}$ but must be capable of delivering $\bar{p}_{g, k}$ in the event of a failure in the system or significant deviation from forecast load values during operation.

Ramping Down A similar approach is taken to the shutting down of slow units. The $v$ and $w$ variables are linked as follows:

$$
w_{g, k+l}+v_{g, k+1} \geq v_{g, k} \quad \forall g \in G, k \in K \mid k+S D T_{g} \leq K, 1 \leq l \leq S D T_{g}
$$


These constraints are valid for $k+S D T_{g} \leq K$ as the model is only concerned with the planning horizon to $K$. They force a unit to shut down over $S D T_{g}$ steps if a slow unit switches off $\left(v_{g, k}=1\right.$ and $\left.v_{g, k+1}=0\right)$.

Ramp down constraints for slow units can then be expressed as:

$$
\bar{p}_{g, k-1} \leq p_{g, k}+S D_{g} S D T_{g}\left(w_{g, k}-w_{g, k-1}\right)+R D_{g} v_{g, k-1} \quad \forall g \in G, k \in K \backslash\{1\}
$$

The available power in time step $k-1$ less the shut down or ramp down rate cannot exceed the output power $p_{g, k}$ at the next step $k$. The slow unit shuts down over $S D T_{g}$ steps after $k$.

Ramp down constraints for fast units are:

$$
\bar{p}_{g, k-1} \leq p_{g, k}+S D_{g}\left(v_{g, k-1}-v_{g, k}\right)+R D_{g} v_{g, k}
$$

A slow unit can only be in any one state (starting up, in production or shutting down) at any time $k$ so the following are valid:

$$
u_{g, k}+v_{g, k}+w_{g, k} \leq 1 \quad \forall g \in G, k \in K
$$

Power output Ramping In addition to the constraints on $\bar{p}_{g, k}$, the difference between the power output in step $k, p_{g, k}$ and the power output in its neighbouring time step must also be bounded.

$$
p_{g, k-1} \leq p_{g, k}+S D_{g}\left(v_{g, k-1}-v_{g, k}\right)+R D_{g} v_{g, k}
$$

A similar constraint adapted for slow units is:

$$
p_{g, k-1} \leq p_{g, k}+S D_{g} S D T_{g}\left(w_{g, k}-w_{g, k-1}\right)+R D_{g} v_{g, k-1} \quad \forall g \in G, k \in K \backslash\{1\}
$$

\subsection{Start Up and Shut Down}

A set of real start up and shutdown variables (bounded by 1) sstart $_{g, k}$, hstart $_{g, k}$ were introduced to simplify implementation of hot and cold start costs, they indicate a unit is cold-started/hot-started in time $k$. As noted in [12], such real variables are helpful in implementing some of the inequalities in UC MIP models and do not substantially impact computational performance.

Start-up Cost It takes time for a unit to warm up before it can be synchronised to the system. It takes more time to reach minimum production limit, $\underline{P}_{g}$. During these times the units incur fuel costs. The longer the unit has been offline, the colder it will be and the more fuel it will require starting up.

In most MILP formulations, the start-up exponential costs are approximated by a simple step function. Is is assumed that the start up cost is triggered when 
any slow unit begins to start up or any fast unit switches state from off to on. For any fast unit $\left(S U T_{g}=1\right)$ the following is valid:

$$
\text { cstart }_{g, k} \geq v_{g, k}-\sum_{n=1}^{t_{g}^{\text {Cold }}+D T_{g}} v_{g, k-n} \quad \forall k \in K \mid k>n, g \in G
$$

In the case of slow units, $S U T_{g}>1$, a unit that begins to start up in time step $k$ will be fully synchronised and ready to deliver power in time $k+S U T_{g}$. The start up cost is assumed to be incurred at time $k+S U T_{g}$ :

$$
\text { cstart }_{g, k+S U T_{g}} \geq u_{g, k}-\sum_{n=1}^{{ }_{t_{g}}^{\text {Cold }}+D T_{g}} u_{g, k-n} \quad \forall k \in K \mid k>n, g \in G
$$

In both cases the summation term is only valid for $k>n$. Conditions for the periods before the planning horizon are handled separately.

The unit is hot-started if online in $k$, offline in $k-1$ and not cold started:

$$
\text { hstart }_{g, k} \geq v_{g, k}-v_{g, k-1}-\text { cstart }_{g, k}
$$

Start-up costs can be captured as:

$$
c_{g, k}^{u} \geq c c_{g}\left(\text { cstart }_{g, k}\right)+h c_{g}\left(\text { hstart }_{g, k}\right)
$$

Start-up costs occur only in time-step $k$ if the generator was offline in timestep $k-1$ and then either hot or cold started in $k$, so the following are also valid: $v_{g, k-1}+$ hstart $_{g, k}+$ cstart $_{g, k} \leq 1$ and hstart $_{g, k}+$ ctart $_{g, k} \leq v_{g, k}$.

Shut-down Cost A traditional thermal generator must lower its output and then de-synchronise from the system before shutting off. During this time, units are using fuel and generating power between minimum generation and zero, therefore incurring a cost.

$$
c_{g, k}^{d} \geq C_{g}^{d}\left(v_{g, k-1}-v_{g, k}\right) \quad \forall g \in G, k \in K
$$

\section{Methodology}

The MILP model is not full dimensional so that a polyhedral analysis is difficult. Strong formulations can be identified by empirical testing on meaningful test instances. The UC MILP model described in Section 3 was implemented in C and solved using XpressMP 7.7 on a Dell 64 bit Windows 8 machine with Intel i5 $3.2 \mathrm{GHz}$ processor and $8 \mathrm{~GB}$ of Ram. The implementation was first tested and verified on the Kazarlis 10 unit system [14].

The MILP model was then tested on UC instances based on the 54 unit Irish system with demand and wind power data for 2014 at a 15 time step. The year was solved on a rolling basis. Each 24 hour period was solved, the system settings 
at the end of the day were used as the initial conditions for the following day. We tested 1) the gross demand and 2) the net demand (the gross demand offset by the available wind). The demand and wind power data for Ireland in 2014 were extracted from [11]. For the purposes of testing, actual wind power (which may have been curtailed at certain times) was used rather than forecasted wind.

The Irish generation system data is derived from the Single Energy Market data available from Ireland's Commission for Energy Regulation, [6]. Figure 3 shows the fuel mix used is 2014. Many units have low min up/down times which provides flexility to integrate wind power. The Irish system has approximately 8,500 MW of conventional power, some pumped storage, no nuclear units, approximately 3,000 MW wind capacity and two HVDC interconnectors to the UK. Hydro-units and pumped storage were removed for the purposes of testing. Initial states were based on the $G U$ s most likely to be online, [6]. Reserve was assumed to be $10 \%$ of demand with more realistic rules to be tested later.

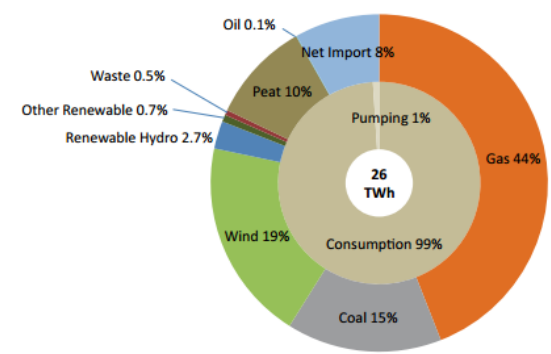

Fig. 3: Fuel mix used in Ireland to satisfy demand in 2014

\section{Results and Analysis}

Missing values of the load data were imputed during pre-processing. Initial analysis of the 2014 load data revealed seasonal, trend and diurnal patterns. The profile of the diurnal pattern is similar across weekdays with only slight weekend variation at weekends as shown in the relative daily demand profiles in Figure 4.a. The amplitude of the profile differs by season with stronger evidence of distance between the seasonal profiles in Figure 4.b which was confirmed using a Euclidean measure.

We also tested fitting an ARIMA model to describe the load data. The data were smoothed and adjusted by the soil temperature at Dublin Airport, the seasonal trend was removed. The ARIMA model could be used in future stochastic programming implementations.

There was evident variability in the wind power data. Our interest in the load and wind data in this paper is to compare the gross load and net load after integrating the wind power. Several similarity or distance measures can be used 
to quantify the similarity/difference between time series data. We calculated the absolute difference between the two series as a simple measure and found an average of 577 MW. Figure 5 shows the distributions of the gross and net loads for comparison.

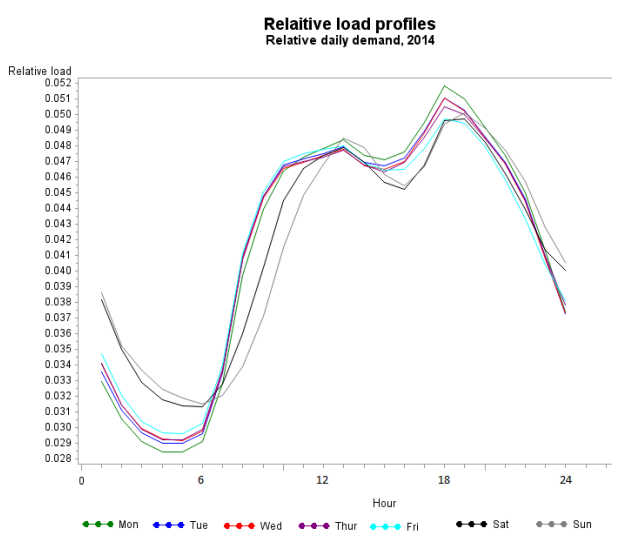

(a) Relative daily demand

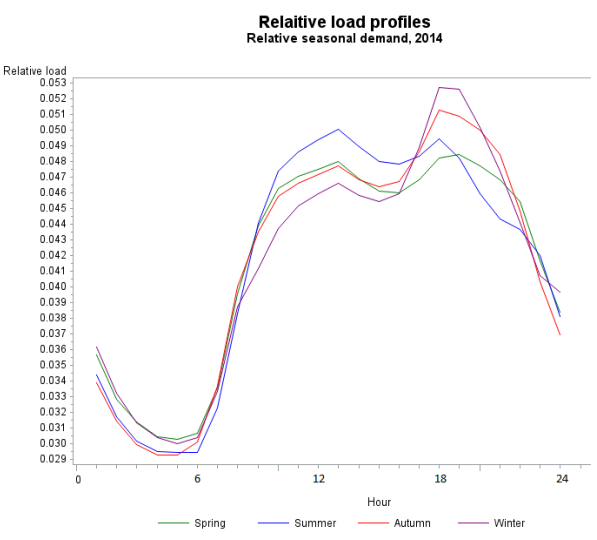

(b) Relative Seasonal demand

Fig. 4: Comparison of daily and seasonal load profiles, Ireland 2014

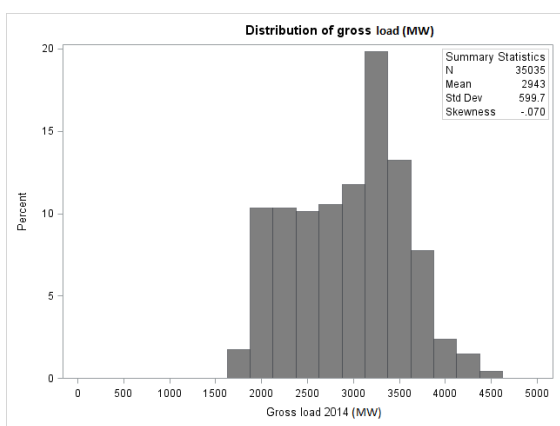

(a) Gross Load (MW)

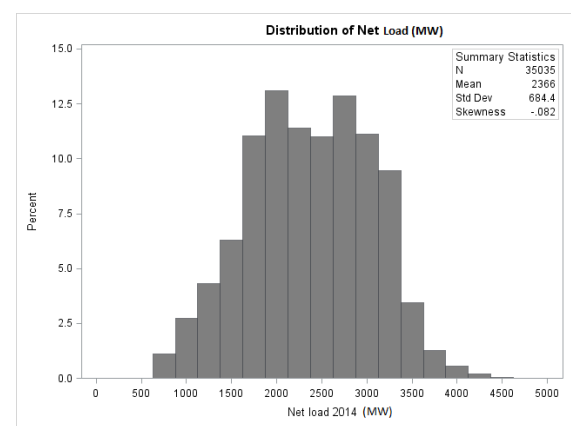

(b) Net Load (MW)

Fig. 5: Distributions of Gross and Net load (MW)

\subsection{MILP Results}

A number of instances based on the test system in [14] were tested. It is not possible to compare these results directly to those published in [14] or [5] as the information given there is incomplete e.g., we have no knowledge of the system 
initial power levels, ramping rates or shutdown costs. These have a significant bearing on the feasibility of problem instances and solution times. $G_{g}$ depends on the initial state of the system. This has particular significance for the practical application of UC MILP models which TSOs use to manage the electricity system on a rolling daily basis. Very small changes to the initial system commitment can make the same load profile become a difficult problem instance.

The MILP model was then tested on the 54 unit Irish test system at a 15 minute time resolution. Results below show the impact of the problem instance variability on solution times. The average solution times for the gross load values i.e., disregarding the wind power, was $70 \mathrm{~s}$ with a standard deviation of $10 \mathrm{~s}$. All instances solved at the root node. The minimum load value of 1,665 MW occurs on a summer night while the maximum demand of 4,614 MW occurs on a winter evening. This is consistent with traditional demand profiles in a temperate climate like Ireland. Figure 6 shows distributions of MILP run times for the gross and net instances.

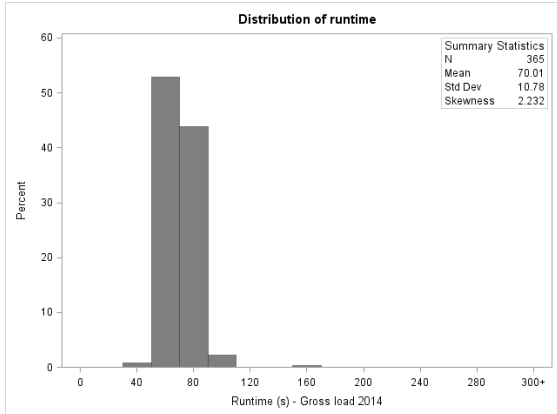

(a) Gross Load instances

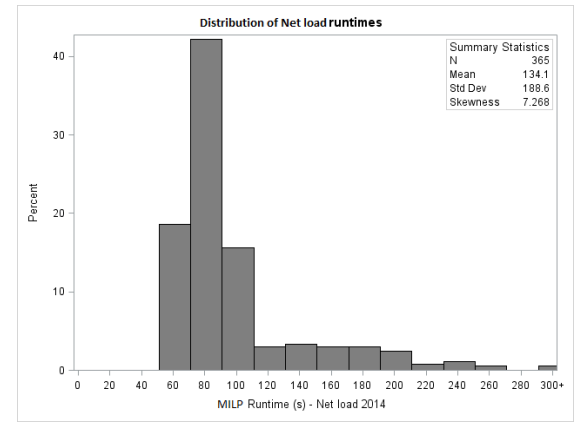

(b) Net Load instances

Fig. 6: MILP Runtime distributions

The net load instances proved more challenging. In these instances the gross demand is offset by the wind power to give a net load instance. The minimum net load value of $642 \mathrm{MW}$ occurs on a winter morning while the maximum net load of 4,487 MW occurs on a winter evening. The depth of the annual net load low gives some indication of the challenges in managing systems with significant RESs. Only five thermal generating units are required to meet this demand. Using the simple $10 \%$ reserve rule highlights the security issue that would arise.

The average solution time of the net load instances was $134 \mathrm{~s}$ with a standard deviation of $187 \mathrm{~s}$. There was an average of 72.3 nodes in the tree although many instances solved quickly at the root node. Figure 7 shows an example of the net load for three consecutive days in January 2014. The instances on either side of $23^{\text {rd }}$ of January solve in 76 and $83 \mathrm{~s}$ respectively at the root node. In contrast the net load of $23^{r d}$ solves in just over 1,024 $\mathrm{s}$ after exploring 3,698 nodes. It can be observed that the more challenging instance has a deeper trough and higher peak. More ramping is required. 
A simple MLR regression model to explain the solution time of a problem instance was tested. The load instances are effectively a set of time series data. Approaches to summarising time series data are described in $[3,16]$. The load data can be represented as a set of summary statistics such as measures of central tendency and variation. In the case of load data, areas such as night time valley, morning peak, evening peak may also be useful in summarising the load instance. In all, 21 possible explanatory variables were identified. A stepwise backward elimination approach was used to identify which were statistically significant in the regression model. The final regression model used only the average down ramp, variance and standard deviation of the load instance $\left(R^{2}\right.$ value of 0.42$)$. A GLM model with an interaction term between the down ramp and variance improved the fit of the final model slightly. While this is not a particularly good model to predict the runtime for this UC model, it is useful for our purpose of identifying what makes a UC instance more difficult to solve. The indications are that problem instances with higher variability are harder to solve. Such load instances require more ramping response from the generation system. It would make sense that the ramping constraints in the MILP are binding for such instances but possibly redundant for less variable instances.

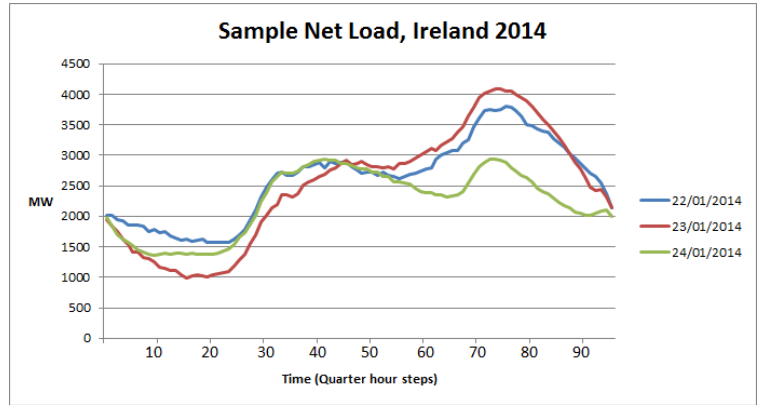

Fig. 7: January 2014

The final integer solutions reported were often found early in the search. The remaining search time was spent improving the Bestbound and reducing the integrality gap. However for some instances, multiple integer solutions with similar objective functions were found which gives an indication of the symmetry problems that can arise. MILP techniques are considered exact approaches. However commercial solvers employ a number of heuristics and cut strategies to improve performance. This suggests that the solver plays a significant role in optimising solution times. The nature and details of the techniques used by the solvers are not generally publicly available so the solver can be treated as a blackbox, with each of the control parameters analogous to a treatment effect with possible interactions. The MILP model was tested in a Design of Experiments framework to evaluate which solver control parameters settings might be beneficial for more challenging UC instances. The control parameters consid- 
ered were presolve, cutstrategy and heurstrategy. The default control parameter settings were most effective in general.

\section{Conclusion}

This paper presents a UC sub-hourly MILP model and demonstrates the model's performance variability on a large set of test instances based on the Irish electricity system. Problem instances where the demand is offset by the available wind power are more challenging to solve and involve more ramping of the generation system. Solutions tend to shut down in the last time $\operatorname{step}(\mathrm{s})$ as the approach does not look forward beyond the 24 hour horizon. The power available during start-up and shut-down of slow units is not directly costed in the objective function but is included in the startup and shut down costs. In the case of the Irish system, the shut down costs are zero so units can be shut down freely. This overly flexible approach to operating the grid may not be desirable. A symmetry problem was noted in the MILP solutions which additional reserve and cycling constraints may reduce. Switching to sub-hourly time steps allows more flexibility in grid operations. A sub-hourly approach not only requires changes to current constraints, but may also require new constraints to better approximate the actual grid operations that could previously be ignored.

\section{Acknowledgements}

The work of Bernard Fortz is supported by the Interuniversity Attraction Poles Programme P7/36 "COMEX" initiated by the Belgian Science Policy Office.

\section{References}

1. Arroyo, J., Conejo, A.: Optimal response of a thermal unit to an electricity spot market. Power Systems, IEEE Transactions on 15(3), 1098-1104 (2000)

2. Bertsimas, D., Litvinov, E., Sun, X., Zhao, J., Zheng, T.: Adaptive robust optimization for the security constrained unit commitment problem. Power Systems, IEEE Transactions on 28(1), 52-63 (Feb 2013)

3. Bickel, P.J., Lehmann, E.L.: Descriptive statistics for nonparametric models i. introduction. The Annals of Statistics 3(5), 1038-1044 (1975)

4. Bienstock, D., Mattia, S.: Using mixed-integer programming to solve power grid blackout problems. Discrete Optimization 4(1), 115 - 141 (2007)

5. Carrion, M., Arroyo, J.: A computationally efficient mixed-integer linear formulation for the thermal unit commitment problem. Power Systems, IEEE Transactions on 21(3), $1371-1378$ (aug 2006)

6. CER: Validated 2011-12 sem generator data parameters. Tech. rep., Commission for Energy Regulation (2011), http://www . allislandproject.org/en/market_decision_documents . aspx?page $=4 \&$ article=151a9561-cef9-47f2-9f48-21f6c62cef34, accessed Nov. 2013

7. Cheng, C.P., Liu, C.W., Liu, C.C.: Unit commitment by lagrangian relaxation and genetic algorithms. Power Systems, IEEE Transactions on 15(2), 707-714 (2000) 
8. Cohen, A.I., Yoshimura, M.: A branch-and-bound algorithm for unit commitment. Power Engineering Review, IEEE PER-3(2), 34-35 (1983)

9. Dillon, T., Edwin, K.W., Kochs, H.D., Taud, R.J.: Integer programming approach to the problem of optimal unit commitment with probabilistic reserve determination. Power Apparatus and Sys, IEEE Trans on PAS-97(6), 2154-2166 (1978)

10. Doherty, R., O'Malley, M.: A new approach to quantify reserve demand in systems with significant installed wind capacity. Power Systems, IEEE Transactions on $20(2), 587-595(2005)$

11. Eirgrid: System performance data (2016), http://smartgriddashboard.eirgrid. com, accessed March 2017

12. Hedman, K., O'Neill, R., Oren, S.: Analyzing valid inequalities of the generation unit commitment problem. In: Power Systems Conference and Exposition, 2009. PSCE '09. IEEE/PES. pp. 1-6 (2009)

13. Juste, K.A., Kita, H., Tanaka, E., Hasegawa, J.: An evolutionary programming solution to the unit commitment problem. Power Systems, IEEE Transactions on 14(4), 1452-1459 (1999)

14. Kazarlis, S., Bakirtzis, A., Petridis, V.: A genetic algorithm solution to the unit commitment problem. Power Systems, IEEE Transactions on 11(1), 83-92 (1996)

15. Kiviluoma, J., Meibom, P., Tuohy, A., Troy, N., Milligan, M., Lange, B., Gibescu, M., O'Malley, M.: Short-term energy balancing with increasing levels of wind energy. Sustainable Energy, IEEE Transactions on 3(4), 769-776 (Oct 2012)

16. McLoughlin, F., Duffy, A., Conlon, M.: Characterising domestic electricity consumption patterns by dwelling and occupant socio-economic variables: An irish case study. Energy and Buildings 48, 240-248 (2012)

17. Merlin, A., Sandrin, P.: A new method for unit commitment at electricite de france. Power Apparatus and Systems, IEEE Transactions on PAS-102(5), 1218 $1225(1983)$

18. Papavasiliou, A., Oren, S.S.: Multiarea stochastic unit commitment for high wind penetration in a transmission constrained network. Operations Research 61(3), 578-592 (2013)

19. Sheble, G., Fahd, G.: Unit commitment literature synopsis. Power Systems, IEEE Transactions on 9(1), 128-135 (1994)

20. Snyder, W.L., Powell, H., Rayburn, J.C.: Dynamic programming approach to unit commitment. Power Systems, IEEE Transactions on 2(2), 339-348 (1987)

21. Takriti, S., Birge, J., Long, E.: A stochastic model for the unit commitment problem. Power Systems, IEEE Transactions on 11(3), 1497-1508 (1996)

22. Ting, T., Rao, M.V.C., Loo, C.: A novel approach for unit commitment problem via an effective hybrid particle swarm optimization. Power Systems, IEEE Transactions on $21(1), 411-418$ (2006)

23. Troy, N., Flynn, D., Milligan, M., O'Malley, M.: Unit commitment with dynamic cycling costs. Power Systems, IEEE Transactions on 27(4), $2196-2205$ (2012)

24. Troy, N., Flynn, D., O'Malley, M.: The importance of sub-hourly modeling with a high penetration of wind generation. In: Power and Energy Society General Meeting, 2012 IEEE. pp. 1-6 (July 2012)

25. Tuohy, A., Meibom, P., Denny, E., O’Malley, M.: Unit commitment for systems with significant wind penetration. Power Systems, IEEE Transactions on 24(2), 592-601 (2009)

26. Zhuang, F., Galiana, F.: Unit commitment by simulated annealing. Power Systems, IEEE Transactions on 5(1), 311-318 (1990)

27. Zhuang, F., Galiana, F.: A more rigorous and practical unit commitment by lagrangian relaxation. Power Systems, IEEE Transactions on 3(2), 763-773 (1988) 